\title{
Enhanced Electrical Model of Lithium-Based Batteries Accounting the Charge Redistribution Effect
}

\author{
Maryam Bahramipanah \\ Ecole Polytechnique Fédérale \\ de Lausanne \\ Lausanne, Switzerland \\ maryam.bahramipanah@epfl.ch
}

\author{
Dimitri Torregrossa \\ Ecole Polytechnique \\ Fédérale de Lausanne \\ Lausanne, Switzerland \\ dimitri.torregrossa@epfl.ch
}

\author{
Rachid Cherkaoui \\ Ecole Polytechnique Fédérale \\ de Lausanne \\ Lausanne, Switzerland \\ rachid.cherkaoui@epfl.ch
}

\author{
Mario Paolone \\ Ecole Polytechnique Fédérale \\ de Lausanne \\ Lausanne, Switzerland \\ mario.paolone@epfl.ch
}

\begin{abstract}
Within the context of the electrical circuit modeling of batteries, this paper proposes an improvement of the most common electric equivalent circuit used for Lithium cells. The main improvement is based on the modeling of the so-called charge redistribution phenomenon that characterizes the dynamic voltage during charging/discharging and relaxation phases. In particular, the aim of the paper is to prove that the model recently proposed by the Authors to represent the same phenomenon in supercapacitors, can be extended also to Lithium batteries. The proposed model is validated by means of experimental results carried out on a $30 \mathrm{Ah} 2.3 \mathrm{~V}$ LithiumTitanate cell with reference to different charge/discharge cycles.
\end{abstract}

Keywords-battery modeling, recovery effect, residual charge, redistribution phenomena

\section{INTRODUCTION}

In the last decades the need and the importance of the energy storage has been increased by the large penetration of distributed generation and renewable energy resources. Among the different type of storage technologies, electrochemical batteries play a key role especially in mobility and distribution network applications (e.g., [1]). In fact these device are characterized by relative high value of energy density, long life duration and, in certain case, a low environmental impact [2].

Within the context of power systems, their typical use refers to transmission and distribution network ancillary services like: peak shaving, voltage control, line congestion management and frequency support (e.g., [1],[3]).

Since each of the above-listed applications is characterized by cycles with different dynamics, charging/discharging phases, delivered currents and duration of rest phases (or relaxation phase), the accurate modeling of the internal dynamics of these devices is of utmost importance.

The study of the literature concerning electric batteries modeling (e.g., [4]) allows to identify three main categories of models, namely: electrochemical models (e.g., [5]-[6]), kinetic battery models (e.g., [7]) and electrical circuit models (e.g., [89]).

Electrochemical models are the most accurate ones since they are based on complex non-linear differential equations aiming at describing the electrochemical processes taking place within the battery cell (in particular, on the electrolyte/electrodes interface). However, this type of models call for a detailed knowledge of the battery chemical processes, expensive instrumentation for analyzing the microspore structure of electrolyte and electrodes, and high computational resources. From this standpoint it seems difficult to use this type of model for real-time battery power management systems [6].

The second category of models, namely the kinetic battery representation [7], is able to depict the so-called non-linear capacity effect of batteries and predict the available Amperehours - Ah - or run-time. These models are based on equations of reduced complexity that model the kinetic process of the chemical reactions within the battery cell. Even if these models are characterized by accurate State-of-Charge $(\mathrm{SoC})$ tracking and run-time prediction, they cannot describe the electrical dynamic (i.e., current and voltage time evolution) that are extremely important for the above applications for which, in general, electric circuit models are preferred.

The third category, the electrical circuit models [9], are based on equivalent electrical circuits aiming at abstracting the physical phenomena taking place inside the battery cell by use of equivalent electrical circuit. However, available electrical circuit models do not completely account for battery non-linear capacity behaviors. This involves an inaccurate prediction of the remaining battery capacity and, consequently, an inaccurate voltage-current time evolutions predictions [10]. The models that have considered these issues (i.e., [11], [12]), are not feasible for real-time applications, such as real-time performance estimation/prediction for batteries power management, still due to their high complexity.

The aim of the work presented in this paper is to provide an improvement of the most common electric equivalent circuit used for Lithium batteries. The main improvement is based on the modeling of the so-called charge redistribution phenomenon that characterizes the dynamic voltage during charging/discharging and relaxation phases. In particular, the aim of the paper is to prove that the model recently proposed in [13] to represent the same phenomenon in supercapacitors, can be extended also to Lithium batteries. In this respect, the paper first discusses the electrochemical processes at the base of the similarities between these two storage systems. Then, it assesses the parameters of the battery electrical circuit by means of a suitable optimization procedure. Finally, the paper proposes the extension of the model proposed by the Authors 
to represent the charge redistribution in supercapacitors to the case of Lithium batteries. The paper is structure as follows. Section II reports a summary on $\mathrm{SoC}$ calculation based on the Columbian method [14], redistribution of charges inside the electrolyte of the cell battery and the electric circuit model. In section III an optimized assessment of the electrical parameters of the most common battery equivalent circuit is described. Then, in section IV, an enhanced model taking into account the redistribution of the charges is deeply described and experimentally validated. The last section concludes the paper by summarizing the main improvement of the proposed model.

\section{BACKGROUND}

This section focuses on describing the models adopted in the paper do define the battery $S o C$ and its internal equivalent circuit. In what follows we will make reference to a LithiumTitanate cell characterized by a 30 Ah rated capacity and a nominal cell voltage of $2.3 \mathrm{~V}$.

\section{A. General formulation of State of Charge}

Generally, the computation of the $S o C$ is the main issue in electrochemical batteries models. There are several techniques to evaluate the $S o C$ [15] (Ampere-hour counting, Kalman filter [16], Artificial Neural Networks [17], online monitoring via battery impulse response [18], [19]). In this work, we have used the method adopted in [14] in which the general equation describing the evolution of $\mathrm{SoC}$ is:

$$
S o C=\frac{C\left(\mathrm{t}_{0}\right)-\alpha(I, \theta) \int_{t_{0}}^{t} i(t) d t}{C(I, \theta)}
$$

Where $\alpha(I, \theta)$ is the efficiency coefficient associated to the battery charge and discharge; $C\left(t_{0}\right)$ is the battery capacity at the initial time $\underline{t}_{0} ; C(I, \theta)$ is the battery capacity for a constant current discharge rate at constant electrolyte temperature $\theta, i(t)$ is the instantaneous current value. The current is assumed to be positive during discharging phase and negative during charging phases. In this work, the temperature $\theta$ is assumed to be constant. It is worth mentioning that (1) does not represent the entire $S o C$ estimation process proposed in [14]. We here report only the main concept and suggest the readers to use [14] for further details.

\section{B. Recovery and rate capacity effects}

As known, electrochemical storage systems exhibit the following behavior: by increasing the load current, the capacity is fading. So, the fact that the higher the discharging current the lower the real capacity, is a known phenomenon called "rate capacity effect". With the term C-rating we henceforth define the capacity for a given time of discharge. As known, it is possible to assume that in the electrolyte there are two layers though which the charges are passing (see Fig. 1) [4]. In particular, the charges are directly delivered toward the load through the surface layer. The inner layer is a storage one that supplies charges only to the surface layer. Consequently it is possible to assume that the $S o C$ represents the amount of the available charges in the surface layer.



Fig. 1. Multiple layer structure of the battery electrolyte.

The exchanging charge between these two layers is the major responsible of the nonlinear behavior of the battery. If the cell is allowed to relax after its discharge, the charges living in the inner layer will come to the surface layer and will become available after a period of time. This nonlinearity characterizes the battery recovery effect. The rate of charge flow between these two layers is henceforth represented with the fixed conductance hereafter indicated with $k$. When a load is connected to the battery, the surface charge will be reduced rapidly and the inner charge will remain inside the battery. When the load is removed, charge would flow from the inner layer to the surface layer to reach an equilibrium. Consequently, the battery open circuit voltage $V_{O C}$ would increase and more charge would be available to the load compared to the case where the battery is continuously connected to the load (in this case the surface charge will drop to zero). Based on the above physical considerations, it is possible to assume that the charges of the inner layers play a key role during the redistribution phase.

In order to illustrate the recovering-redistribution phases and the rate capacity effects, Fig. 2 illustrates the time evolution of the targeted battery cell voltage $\left(V_{C E L L}\right)$ with $0.6 \mathrm{C}$, $1 \mathrm{C}$, and $1.5 \mathrm{C}$ charging/discharging rates. As it can be observed, when the cell current flow is stopped, the redistribution and the associated recovery effect take place and the $V_{C E L L}$ increases $(300<\mathrm{t}<900)$. Also, the rate capacity effect can be clearly seen in the discharging phases of Fig. 2 (i.e., when the discharging rate decreases, the battery has more potential to reach the equilibrium). Indeed, after having discharged the battery with higher currents, larger amount of charges can be recovered.


Fig. 2. Time evolution of the targeted Lithium cell voltage with different discharging rates.

\section{Battery electrical model}

The most common electrical model of Lithium electrochemical batteries is that one based on equivalent Thevenin-circuit [20],[21]. This models is available in three 
different configurations: internal resistance (IR) model, one time constant (OTC) model and two time constant (TTC) model [22]. As it is shown in [22], the use of two $R C$ time constant model (shown in Fig. 3) allows to take into account slow electrochemical process characterizing the slow charging/discharging and redistribution phases. This model represents the best tradeoff between accuracy and complexity. In particular, it is composed by the following parameters:

- $R_{0}$ as the battery input resistance which is used to characterize the charge/discharge energy losses of the battery cell;

- $R_{S}, C_{S}$ as the resistance and the capacitance of the first branch respectively, modeling the fastest electric dynamics;

- $\quad R_{L}, C_{L}$ as the resistance and the capacitance of the second branch respectively, modeling the slowest electric dynamics;

- $\quad E_{m}$ as the electromotive force of the battery which can be measured as voltage across the open circuit terminals.

All the above parameters are not linear and depend to the SoC and temperature of the cell as well [23].

In the work here proposed the temperature effect on the electric parameter is not accounted since all the test have been performed into a climatic chamber where the temperature is strictly set to the nominal value of the cell, namely $25^{\circ} \mathrm{C}$.

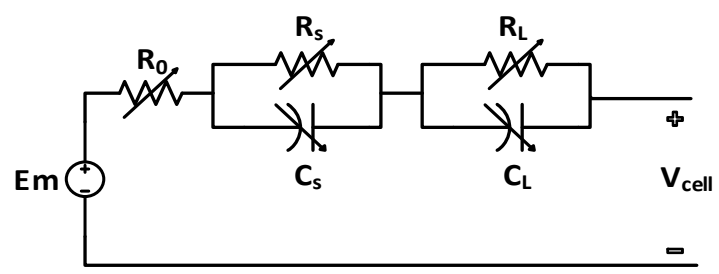

Fig. 3. Typical TTC model for Lithium cells.

\section{OPTIMAL ASSESSMENT OF CELL BRANCHES PARAMETERS}

Several work have been performed for estimating the parameters of the cell and modeling their dependency to the SoC. Despite of being successful in many aspects, most of them still have several drawbacks. For instance, for modeling the non-linear open circuit voltage, $E_{m}(S o C)$, in [21] the Authors employed a sophisticated electrical network extracted from physical process. The main drawback of such an approach is that it requires a non-negligible computational overhead. Additionally, in [24], the model is just valid for timescales ranging from milliseconds up to a few seconds at a constant SoC and temperature.

In the work here proposed the assessment of the parameters of the circuit of Fig. 3 is performed by using experimental tests characterized by different sub-phases: constant charging phase, constant discharging phase, and relaxation phases with $1 \mathrm{C}$ discharging rate (see Fig. 4).
It is possible to assume that, during the targeted cycle, the cell voltage, $V_{C E L L}$, can be represented as a function of $\mathrm{SoC}$ and the relevant circuit parameters of Fig. 3:

$$
\begin{aligned}
& v_{\text {cell }}(t)=f_{1}\left[i(t), \operatorname{SoC}, R_{S i}, C_{S i}\right]+f_{2}\left[i(t), \operatorname{SoC}, R_{L i}, C_{L i}\right] \\
& +f_{2}\left[i(t), \operatorname{SoC}, R_{0 i}, E_{m i}\right]
\end{aligned}
$$

Where:

- $f_{i}(i=1,2,3)$ are the circuit equations of the cell model and represent the contribution of electrical elements to $v_{\text {cell }}(t)$;

- $\quad R_{S i}, C_{S i}, R_{L i}, C_{L i}, R_{0 i}, E_{m i}$ are $i^{\text {th }}$ element of the piecewise approximation related to the non-linear capacitances and resistance of the cell model (see Fig. 3).

The evaluation of the electrical parameters of equation (2) has been performed by following a modified procedure that was proposed in [13]. In particular, the variation of $\mathrm{SoC}$ has been divided into a certain number of intervals (in our case 11). For each of these intervals a first set of the battery parameters has been evaluated by using the procedure given in [8]. Then, in order to improve the values of these parameters, a least square fitting procedure has been used. It is therefore possible to define the following optimal problems for inferring the model parameters by comparing the $v_{\text {cell }}$ given by the battery and the measured one, $v_{\text {cell, } m}$ :

$$
\begin{aligned}
& \underset{R_{s}, C_{s}, R_{L}, C_{L}, R_{0}, E_{m}}{\arg \min }\left\{v_{c e l l, m i}(t)-f_{1}\left[i(t), S o C, R_{S i}, C_{S i}\right]\right. \\
& \left.-f_{2}\left[i(t), S o C, R_{L i}, C_{L i}\right]-f_{2}\left[i(t), S o C, R_{0 i}, E_{m i}\right]\right\}^{2}
\end{aligned}
$$

The trend of $v_{\text {cell }}(t)$ with the optimally-identified parameters is shown in Fig. 4. It can be observed that the optimallyidentified parameters allows to obtain a quite good matching between the simulated $v_{\text {cell }}(t)$ and the measured one.

However, even with optimally-identified parameters, the circuit of Fig. 3 cannot accurately reproduce the timeevaluation of the battery $D C$ voltage if cycles other than the ones used to infer the parameters are considered. In order to show such a limitation, we used the same cycle as the one that was used for estimating the parameters (Fig. 4) but with different values C-rate. In Fig. 5, the time evolution of battery $D C$ voltage with $20 \mathrm{~A}(0.66 \mathrm{C})$ discharging current obtained from the simulation is compared with the measured one. It can be clearly seen that in this case the TTC model, with the optimally-identified parameters related to the cycle of Fig. 4, is unable to consider the flow of charges coming from the inner layer to the surface layer and vice versa.

\section{BATTERY CHARACTERISTICS AND PROPOSED BATTERY MODEL}

This section discusses the proposed enhanced battery model that, by accounting the charge redistribution effects, allows solving the mismatch between the battery model behaviors and the experimental ones when the battery is subjected to cycles different from the one used to identify its internal parameters. 

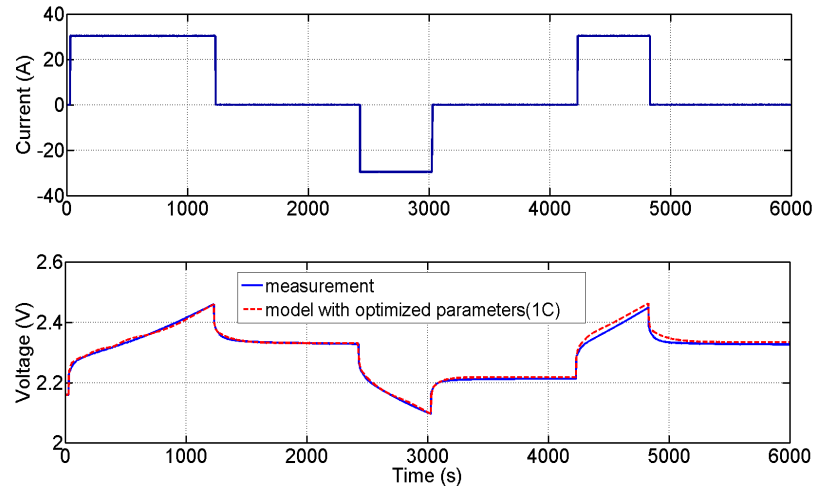

Fig. 4. Comparison of simulated and measured trend of the Lithium cell voltage for the case of optimally-identified parameters.
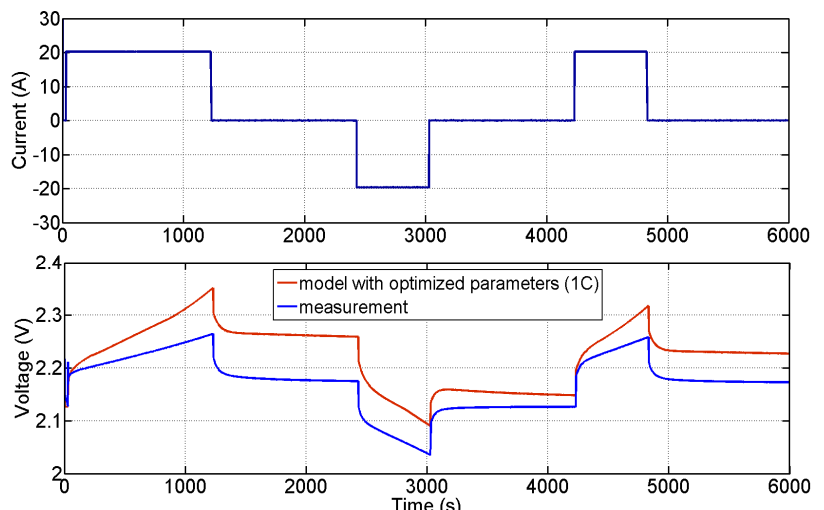

Fig. 5. Comparison of simulated and measured trend of the Lithium cell voltage for the case of optimally-identified parameters obtained from Fig. 4 but with a modified discharge rate $(0.66 \mathrm{C})$.

Before discussing the proposed model, it is worth mentioning that the literature on the subject has already treated this aspect. In particular, an enhanced circuit based model was developed in [11] and [12] by mixing an electrical circuit model [10] with kinetic model [25] to include the battery recovery effect. However, due to the high complexity of this model, it does not appear feasible for applications with low overhead computational means (e.g., real-time performance estimation/prediction of battery power management systems).

On the other hands, in [13] a simple enhanced model accounting the redistribution phenomena in supercapacitors has been proposed. In what follows the same approach is extended to the case of batteries in order to improve the existing TTC model.

\section{A. Computation of the whole parameters model.}

The initial battery capacity, with zero battery current condition maintained for a few hours, is based on the correlation between battery open circuit voltage and the electrolyte density in the assumption that appropriate use/maintenance of the battery has been always granted.

In order to find the value of the $C_{S}$ and $C_{L}$ initial voltages, the initial $S o C$ should be known (starting point). However, the only value that is measurable from the Lithium cell is its terminal voltage (i.e., the cell is open circuit voltage, $V_{O C}$, before the battery cycling). So, in order to find the relationship between initial $S o C$ and $V_{O C}$, a set of tests has been performed. In this respect, we first need to make sure that the battery is fully discharged (i.e., it has no residual charge when the minimum voltage has reached). For this purpose, the battery is discharged with two different sub-phases: i) constant current discharge phase with $1 \mathrm{C}$-rate until reaching its minimum voltage (i.e., $1.73 \mathrm{~V}$ ); ii) constant voltage discharge (the discharge voltage is equal to the minimum battery voltage) until the current reaches $20 \mathrm{~mA}$ (this last vale corresponds to the noise of the measurement system). From this starting point the battery has been charged with constant current value $1 \mathrm{C}$ rate until reaching eight different final voltages $\left(V_{F}\right)$. Then the power generator is turned off and the battery is allowed to rest for two hours. The $V_{\text {cell }}$ reached at this moment is $V_{O C}$ of Table 1. Then the battery is discharged completely as described. The charge extracted during this discharging phase has been evaluated and it represents the amount of total charge stored in the battery. Table I shows the relation between $V_{O C}$ and the available charge inside the battery. For each $V_{O C}$ the associated $S o C$ has been evaluated by dividing the extracted charge by the rate capacity. The nonlinear link between $S o C$ and $V_{O C}$ is shown in Fig. 6.

TABLE I. $\quad V_{O C}$ AND ASSOCIATED STORED CHARGE

\begin{tabular}{|c|c|}
\hline$V_{\text {OC }}(\boldsymbol{V})$ & Charge $($ Ah $)$ \\
\hline 1.77 & 0.03 \\
\hline 1.85 & 0.44 \\
\hline 2.06 & 8.27 \\
\hline 2.18 & 18.27 \\
\hline 2.28 & 23.77 \\
\hline 2.37 & 27.58 \\
\hline 2.46 & 31.50 \\
\hline 2.70 & 35.13 \\
\hline
\end{tabular}

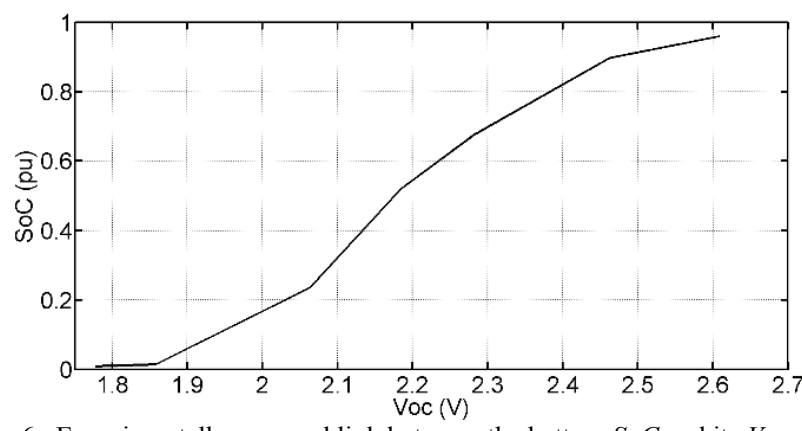

Fig. 6. Experimentally-assessed link between the battery $S o C$ and its $V_{O C}$.

Once the initial $S o C$ is known, it is possible to compute the six parameters $R_{0}, R_{S}, C_{S}, R_{L}, C_{L}$ and $E_{m}$.

As a result, based on the circuit theory, the initial value of the capacitor's voltage $\left(V_{S}\right.$ and $\left.V_{L}\right)$ can be inferred from the Laplace transformation. Thus, the initial states of the battery can be determined by solving equations (4) and (5) (in (5) the $V_{\text {cell }}$ represents the terminal dc voltage of the battery which is $V_{O C}$ for starting point)

$\frac{V_{s}}{V_{L}}=\frac{C_{L} \cdot S+1 / R_{L}}{C_{s} \cdot S+1 / R_{s}}$ 


$$
V_{s}+V_{L}=V_{\text {cell }}-E_{m}
$$

\section{B. Virtual current source for the representation of the charge redistribution.}

As previously mentioned, the model proposed in this paper (schematically represented in Fig. 7) is an extension of what proposed by the Authors in [13] to represent the charge redistribution in supercapacitors. Since both devices, batteries and supercapacitors, are electrochemical energy storage devices characterized by internal flow charge, from inner layer to surface layer and vice versa in battery and from micro-pore to meso-pore and vice versa in supercapacitor, the same modeling proposed in [13] can be applied, in principles, to batteries. It represents an enhanced battery model considering both recovery and rate capacity effects inside the battery. The key point of this model is that it is able to represent the redistribution of the residual charge by using a simple virtual current generator. The optimal model parameter determined earlier allows to accurately model the dynamic behavior of the targeted cell charged and discharged at 1C-rate $\left(I_{\text {rate }}\right)$ along with redistribution phase (Fig. 4). For this particular cycle, the proposed model is already taking into account the internal charge flow between inner and surface layers. So, we consider this case as the reference one. If the C-rate changes, the associated internal flows charges are not the same as those ones evaluated for the 1C-rate cycle. As a result, the idea is to take into account, by using a virtual current generator, the different internal flow charge compared to the reference cycle (1C-rate). In other words, the virtual current generator is used to model the charge flow inside the battery between the inner and surface layers ${ }^{1}$. It is active only when charges are transferring from the two layers of Fig. 1 and with time constants larger than some thousands of seconds. It is worth noting that this virtual current generator does not violate the charge balance of the battery since it is not injecting an external current. In this respect, the principle of charge balance has been numerically validated during the different model validations. It is important to underline that during fast-time varying charges/discharges, the charges available on the surface layer are those mostly used. As a consequence, for these conditions, existing TTC model works, as expected, pretty well. In view of the above, the contribution of the model here proposed is to fulfill the above-identified limitations of existing battery models.

Following the approach proposed in [13], this virtual generator will deliver the following current (note that since the battery model is composed by two RC branches plus $E_{\mathrm{m}}$, in agreement with [13], the virtual current generator has to account three time constants):

$$
I_{v i r}=\frac{Q_{R E D}}{\tau_{S}} \eta_{1} e^{-t / \tau_{S}}+\frac{Q_{R E D}}{\tau_{L}} \eta_{2} e^{-t / \tau_{L}}+\frac{Q_{R E D}}{T} \eta_{3} e^{-t / \mathrm{T}}
$$

Where:

- $Q_{R E D}$ is the amount of charge to be redistributed in order to model the rate capacity and recovery effect;

$$
-\tau_{S}=R_{S} \cdot C_{S}
$$

\footnotetext{
${ }^{1}$ It is important to underline that this flow does not involve any charge flow with the external power supply / load.
}

- $\tau_{L}=R_{L} \cdot C_{L}$

- $\quad T$ is the charging/discharging time;

- $\quad \eta_{1}, \eta_{2}$ and $\eta_{3}$ define the share of each time constant. They are proportional to their respective time constants. So, the third exponential part in equation (6) is associated to $E_{m}$.

Fig. 7. The proposed modified TTC model.

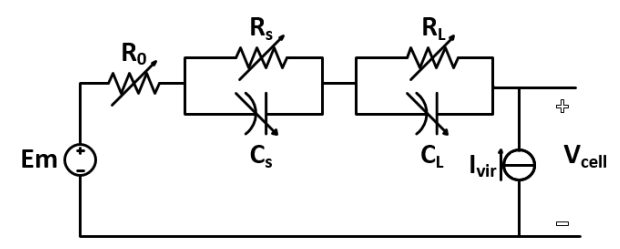

As mentioned, 1C-rate discharging is considered as the dynamic cycle reference.

Let replace the quantity $Q_{R e f}$ with $\Delta Q_{r e f}$ as the amount of charge flowing from the surface to the inner layer or vice versa for each charging/discharging/resting during the cycle at $1 \mathrm{C}$ rating. The difference between this charge $\Delta Q_{\text {ref }}$ and the transferred charge with another discharging rate, is the amount of charge that should be redistributed, $Q_{R E D}$.

For sake of clarity, the calculation of the parameters of the proposed virtual current generator parameters is detailed for the first three sub-phases of the cycle shown in Fig. 5.

First sub phase (charging phase): the initial voltage $V_{0}$ is $2.15 \mathrm{~V}$. By using the values in Table I, the stored charge inside the battery has the value $Q_{\text {init }}$. The associated initial $S o C$ will be obtained by the following equation:

$$
S o C_{\text {init }}=\frac{Q_{\text {init }}}{C\left(\left.I\right|_{1 C}\right)}
$$

Where $C\left(\left.I\right|_{1 C}\right)$ is the rated capacity of the battery corresponding to $1 \mathrm{C}$-rate. The $S o C_{i n i t}$ corresponding to $C\left(\left.I\right|_{1 C}\right)$ is 0.439 . The quantity of charge, $\left.\Delta Q\right|_{1 C}$, that the power generator has to deliver in order to reach the final charge voltage $\left(V_{F}\right)$ with $1 \mathrm{C}$-rate should be determined (as a reference case for charging). In our case $V_{F}$ is 2.264. In order to obtain $\left.\Delta Q\right|_{1 C}$, the $S o C$ of the final point of charging $\left(S o C_{F}\right)$ should be known. For this purpose, Fig. 6 can be used to evaluate $S_{o} C_{F}$. By knowing $V_{F}$, it is possible to compute $V_{O C}$. Table II shows the relation between $V_{O C}$ subsequent to a charging or discharging phase with a same value of $V_{F}$ obtained by experimental tests. It's worth noting that, after a charging phase, the subsequent redistribution phase involves a decay voltage (i.e., $V_{C E L L}$ is decreasing). Conversely, after a discharging phase there is an increase of $V_{C E L L}$. This table is extremely important since, based on the knowledge of $V_{F}$ (during charging/discharging phases), it allows to evaluate the associated $V_{O C}$ and consequently the associated $\mathrm{SoC}$.

Once the two $S o C_{i n i t}$ and $S o C_{F}$ are known, it is possible to evaluate the quantity of charge that the power generator has to deliver in order to reach the final $S o C$ with $1 \mathrm{C}$-rate for the 
reference case. This amount of charge called $\left.\Delta Q\right|_{1 C}$ is equal to:

$$
\left.\Delta Q\right|_{1 C}=\left(S o C_{F}-S o C_{\text {init }}\right) \cdot C\left(\left.I\right|_{1 C}\right)
$$

TABLE II. RELATION BETWEEN $V_{F}$ AND $V_{O C}$ SUBSEQUENT TO CHARGE OR DISCHARGE PHASES.

\begin{tabular}{|c|c|c|}
\hline \multicolumn{3}{|c|}{$\begin{array}{c}\text { Relation between reached final voltage } V_{F} \\
\text { and } V_{O C}\end{array}$} \\
\hline$V_{F}(V)$ & $V_{O C}(V)$ charge & $\begin{array}{c}V_{O C}(V) \\
\text { discharge }\end{array}$ \\
\hline 1.9 & 1.77 & 2.09 \\
\hline 2.0 & 1.85 & 2.15 \\
\hline 2.1 & 2.06 & 2.23 \\
\hline 2.2 & 2.18 & 2.33 \\
\hline 2.3 & 2.28 & 2.43 \\
\hline 2.4 & 2.37 & 2.53 \\
\hline 2.5 & 2.46 & 2.64 \\
\hline 2.7 & 2.60 & - \\
\hline
\end{tabular}

In the targeted cycle we were using a discharge rate of $0.66 \mathrm{C}$. So, in order to take into account the rate capacity effect during this sub phase, it is needed to evaluate the difference of charge between $0.66 \mathrm{C}$-rate $\left(\left.\Delta Q\right|_{0.66 C}\right)$ and the reference cycle. So, $\left.\Delta Q\right|_{C_{i}}$ can be obtained by measuring the charge during the charging phase. So, we have:

$\left.\Delta Q\right|_{C_{i}}=\int_{t_{1}}^{t_{2}} i(t) d t$

Therefore, since the virtual generator has to redistribute the different amount of charge compared to the reference cycle, $Q_{R E D}$ can be easily obtained as:

$Q_{R E D}=\left.\Delta Q\right|_{1 C}-\left.\Delta Q\right|_{C_{i}}$

So for this targeted case we have:

$Q_{R E D}=\left.\Delta Q\right|_{1 C}-\left.\Delta Q\right|_{0.6 C}$

This amount of charge defined by (11) is that one the virtual current generator has to redistribute during the charging phase. During the charging phase there is a higher amount of charge flowing from the surface layer toward the inner layer if the battery is charged with lower current.

Second sub phase (resting phase): during the resting phase, since the power generator is off, the $S o C$ is the same of that one evaluated at the end of the previous sub-phase $\left(S o C_{\text {resting }}\right)$. During this sub phase the recovery effect is taking place.

For the reference case with $1 \mathrm{C}$-rate, $\left.\Delta Q\right|_{1 C}$ is:

$$
\left.\Delta Q\right|_{1 C}=S o C_{\text {resting }} \cdot C\left(\left.I\right|_{1 C}\right)
$$

But, in our case the battery has been charged with $0.66 \mathrm{C}$ rate which make the resting phase different from the reference case. In order to represent this behavior, $\left.\Delta Q\right|_{C_{i}}$ is calculated considering the total charging stored into the battery in the previous charging phase.

$$
\left.\Delta Q\right|_{C_{i}(\text { resting })}=S o C_{\text {init (ch arge-phase })} \cdot C\left(\left.I\right|_{C_{i}}\right)+\left.\Delta Q\right|_{C_{i}(\text { ch arge-phase })}
$$

The difference between these two amounts of charge has to be redistributed during this targeted resting phase. This difference is in reality flowing from surface layer toward inner layer. So the $Q_{R E D}$ is given by (10). And for the targeted subphase it is calculated by (11).

Subsequent phases: For the next sub-phase, the discharging phase, the procedure to be followed is the same one as that one described by (10). The change is that in this sub-phase the charge is flowing from the inner layer to the surface layer. The same consideration can be made for the resting phase subsequent to a discharge phase.

This amount of charge $\left(Q_{R E D}\right)$ is redistributed by the means of the proposed virtual generator described in eq. (6).

In order to assess the limitations of the proposed model, we have investigated its applicability with respect to different discharge rates. As expected, the presented model is not valid with extremely low discharging rates (i.e., less than 4 Amperes in the targeted battery). In fact, the increase of the electrolyte temperature is lower with respect to the reference case illustrated before. The consequences of this lower electrolyte temperature are: (i) its higher viscosity and (ii) its lower ion mobility. This involves that the charge flow on the battery layers is reduced and it cannot be taken into account by the virtual generators here proposed.

\section{MOdel VALIDATION}

\section{A. Experimental set-up}

The proposed model has been experimentally validated with various cycles characterized by different charging, discharging and relaxation phases. The battery specifications are given in Table III.

\section{TABLE III. TARGETED LITHIUM-TITANATE BATTERY SPECIFICATIONS}

\begin{tabular}{|l|c|}
\hline \multicolumn{2}{|c|}{ Lithium-Titanate battery technical data } \\
\hline Nominal capacity & $30 \mathrm{Ah}$ \\
\hline Nominal Energy & $70 \mathrm{Wh}$ \\
\hline Nominal voltage & $2.3 \mathrm{~V}$ \\
\hline Energy density & $63 \mathrm{Wh} / \mathrm{kg}$ \\
\hline Power density & $150 \mathrm{~W} / \mathrm{kg}$ \\
\hline
\end{tabular}

The charging system was controlled by a power supply EAPS 8080-120. The device allowed charging with a completely programmable input voltage and current profile, and it is fully remote controlled.

The discharging system was controlled by Electronic load EA-EL 9080-200. The device allowed for discharging the cell under test in constant current mode, constant voltage mode, and constant power mode. It is also fully remote controlled.

The battery voltage and current have been directly measured by using a 16-bits National Instrument, A/D 9215 conversion cards coupled with voltage sensor current sensor. 
The temperature is kept constant during the test via a climatic chamber.

Fig. 8 compares the terminal voltage responses obtained from simulation using TTC model and the proposed model with experimental results for $0.66 \mathrm{C}$-rate with the same cycle used for estimating the parameters. It can be seen that the proposed model can accurately reproduce the battery dynamic behavior. Furthermore, the model is able to accurately trace the $S o C$ variation. It should be underlined that the mean squared error between the battery measured voltage and the proposed one is extremely low and in the order of $10^{-4}$ and a corresponding value of 0.003 has been evaluated for TTC model.

Fig. 9 compares the simulation and experimental results with another cycle with $0.5 \mathrm{C}$-rate different of the ones used to infer the parameters and a different starting voltage value. It is possible to observe that the proposed model allows modeling the rate capacity and recovery effects of the battery. It is possible as well to observe that if the starting point, initial $V_{O C}$ and/or initial $\mathrm{SoC}$ change, the optimized model shown in Fig. 3 is not able to accurately predict the electrical battery dynamic.

In order to prove the applicability of the model with respect to more realistic charge/discharge profiles, we have adopted the battery current profile inferred from [14] that makes reference to a microgrid application of a battery storage system. In particular, in [14] a specific microgrid was used to assess the performances of a dedicated centralized controller. We have here adopted the battery current profile related to test (a) of [14] and reproduced it by means of the same battery test bench. In Fig. 10 we report the time evolution of the battery DC voltage with respect to this specific test condition. As it can be noticed, even for this peculiar cycle, the proposed model matches the measurements with a high level of fidelity.

\section{CONCLUSION}

Modeling of electrochemical storage systems is a subject that always receives attention in view of the increasing importance of storage devices in power systems and mobility applications. Among the several approaches proposed in the literature to reproduce the dynamic behavior of batteries, the equivalent electrical circuit one is certainly the most popular in view of its low computational overhead and straightforward implementation in electrical circuit solvers. However, this category of models does not entirely account for the battery non-linear capacity behavior. This is due to the inaccurate prediction of the remaining battery charge together with its redistribution. Consequently, an inaccurate voltage-current time evolution prediction are generally obtained.

In this respect, the paper has proposed to enhance the above-mentioned circuit model by representing the so-called charge redistribution phenomenon that characterizes the dynamic of the battery voltage during long time charging/discharging and relaxation phases. In particular, the contribution of the paper has been to prove the feasible extension to the case of Lithium batteries of the model recently proposed by the Authors to represent the redistribution charge phenomenon in supercapacitors.
By making reference to experimental tests carried out on a $30 \mathrm{Ah}, 2.3 \mathrm{~V}$ Lithium-Titanate battery, the paper has first discussed the optimal parameter assessment of the relevant TTC circuit model and, then, it has shown the improvement of the battery model behavior by accounting the charge redistribution phenomenon. In this respect, it can be concluded that the proposed enhanced battery model represents an adequate extension of the classical electrical circuit ones with a minimal computational overhead.



Fig. 8. Time evolution of battery dc voltage with $0.6 \mathrm{C}$
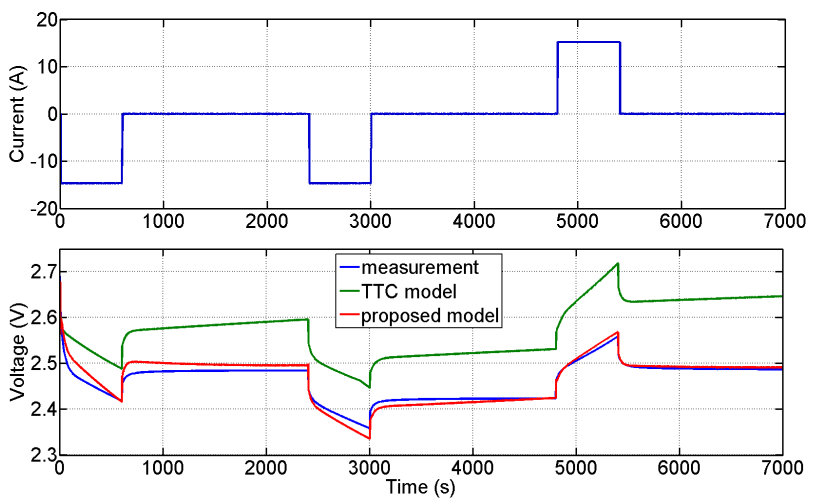

Fig. 9. Time evolution of battery de voltage with $0.5 \mathrm{C}$.
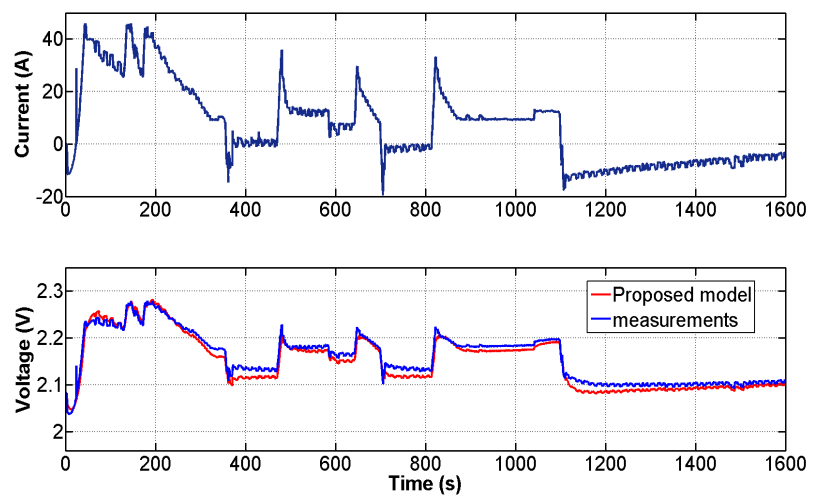

Fig. 10. Time evolution of battery dc voltage with respect to realistic fast charge/discharge profiles.

\section{REFERENCES}

[1] Cigré Technical Brochure, "Electric Energy Storage Systems," Work. Gr. C6.15, no. April, 2011.

[2] A. R. Sparacino, S. M. Ieee, G. F. Reed, R. J. Kerestes, Z. T. Smith, and S. Member, "Survey of Battery Energy Storage Systems and Modeling 
Techniques," in IEEE Power and Energy Society General Meeting, 2012, pp. 1-8.

[3] A. Oudalov, C. Yuen, and M. Holmberg, "Energy Storage is a Key Smart Grid Element," in Cigré Symposium The Electric Power System of the Future, 2011.

[4] B. R. H. M.R. Jongerden, "Which battery model to use?," UKPE 2008, Imp. Coll. London, DTR08-9, pp. 76-88, 2008.

[5] R. Klein, N. a. Chaturvedi, J. Christensen, J. Ahmed, R. Findeisen, and A. Kojic, "Electrochemical Model Based Observer Design for a Lithium-Ion Battery," IEEE Trans. Control Syst. Technol., vol. 21, no. 2, pp. 289-301, Mar. 2013.

[6] D. W. Dees, V. S. Battaglia, and A. Bélanger, "Electrochemical modeling of lithium polymer batteries," J. Power Sources, vol. 110, no. 2, pp. 310-320, Aug. 2002.

[7] J. F. Manwell and J. G. McGowan, "Lead acid battery storage model for hybrid energy systems," Sol. Energy, vol. 50, no. 5, pp. 399-405, May 1993.

[8] S. Abu-Sharkh and D. Doerffel, "Rapid test and non-linear model characterisation of solid-state lithium-ion batteries," J. Power Sources, vol. 130, no. 1-2, pp. 266-274, May 2004.

[9] L. Benini, G. Castelli, a. Macii, E. Macii, M. Poncino, and R. Scarsi, "Discrete-time battery models for system-level low-power design," IEEE Trans. Very Large Scale Integr. Syst., vol. 9, no. 5, pp. 630-640, Oct. 2001.

[10] G. A. R.-M. Min Chen, "Accurate Electrical Battery Model Capable of Predicting Runtime and I-V Performance," IEEE Trans. Energy Convers., vol. 21, no. 2, pp. 504-511, 2006.

[11] J. Zhang, S. Ci, H. Sharif, and M. Alahmad, "An enhanced circuit-based model for single-cell battery," in 2010 Twenty-Fifth Annual IEEE Applied Power Electronics Conference and Exposition (APEC), 2010, pp. 672-675.

[12] J. Zhang, S. Member, S. Ci, S. Member, H. Sharif, and M. Alahmad, "Modeling Discharge Behavior of Multicell Battery," IEEE Trans. ENERGY Convers., vol. 25, no. 4, pp. 1133-1141, 2010.

[13] D. Torregrossa, M. Bahramipanah, E. Namor, R. Cherkaoui, S. Member, and M. Paolone, "Improvement of Dynamic Modeling of Supercapacitor by Residual Charge Effect Estimation," IEEE Trans. Ind. Electron., vol. 61, no. 3, pp. 1345-1354, 2014.

[14] B. Belvedere, M. Bianchi, A. Borghetti, S. Member, C. A. Nucci, M. Paolone, and A. Peretto, "A Microcontroller-Based Power Management
System for Standalone Microgrids With Hybrid Power Supply," IEEE Trans. Sustain. ENERGY, vol. 3, no. 3, pp. 422-431, 2012.

[15] S. Piller, M. Perrin, and A. Jossen, "Methods for state-of-charge determination and their applications," J. Power Sources, vol. 96, no. 1, pp. 113-120, Jun. 2001.

[16] F. Zhang, G. Liu, and M. Ieee, "A battery State of Charge estimation method with extended Kalman filter," IEEE/ASME Int. Conf. Adv. Intell. Mechatronics, pp. 1008-1013, Jul. 2008.

[17] C. . Chan, E. W. . Lo, and S. Weixiang, "The available capacity computation model based on artificial neural network for lead-acid batteries in electric vehicles," J. Power Sources, vol. 87, no. 1-2, pp. 201-204, Apr. 2000.

[18] A. Banaei and B. Fahimi, "Real time condition monitoring in Li-Ion batteries via battery impulse response," IEEE Veh. Power Propuls. Conf., pp. 1-6, Sep. 2010.

[19] A. H. Ranjbar, A. Banaei, A. Khoobroo, and B. Fahimi, "Online Estimation of State of Charge in Li-Ion," IEEE Trans. Smart Grid, vol. 3, no. 1, pp. 360-367, 2012.

[20] Z. M. Salameh, M. a. Casacca, and W. a. Lynch, "A mathematical model for lead-acid batteries," IEEE Trans. Energy Convers., vol. 7, no. 1, pp. 93-98, Mar. 1992.

[21] M. C. Glass, "BATTERY ELECTROCHEMICAL NONLINEAR/DYNAMIC SPICE MODEL," in 31st Intersociety Energy Conversion Engineering Conference, IECEC 96., 1996, pp. 292297.

[22] A. Rahmoun and H. Biechl, "Modelling of Li-ion batteries using equivalent circuit diagrams," PRZEGLACD ELEKTROTECHNICZNY (Electrical Rev. ISSN 0033-2097, R. 88 NR 7b, vol. 2, no. 7, pp. 152$156,2012$.

[23] T. Huria, M. Ceraolo, J. Gazzarri, and R. Jackey, "High Fidelity Electrical Model with Thermal Dependence for Characterization and Simulation of High Power Lithium Battery Cells," in IEEE International Electric Vehicle Conference (IEVC), 2012, pp. 1-8.

[24] B. Schweighofer, K. M. Raab, G. Brasseur, and S. Member, "Modeling of High Power Automotive Batteries by the Use of an Automated Test System," IEEE Trans. Instrum. Meas., vol. 52, no. 4, pp. 1087-1091, 2003.

[25] D. N. Rakhmatov and S. B. K. Vrudhula, "An analytical high-level battery model for use in energy management of portable electronic systems," IEEE/ACM Int. Conf. Comput. Aided Des. ICCAD 2001. IEEE/ACM Dig. Tech. Pap. (Cat. No.01CH37281), pp. 488-493, 2001. 